\title{
Hausse des primes d'assurance-maladie: seuil de tolérance atteint!
}

La poursuite invariable de la hausse des primes chaque automne a déclenché des manifestations d'exaspération qui vont bien au-delà de la mauvaise humeur habituelle dans ces circonstances. De toute évidence ce processus ne peut continuer comme cela. Les atermoiements et les blocages politiques sont incompréhensibles. Les temps du renvoi mutuel de la patate chaude sont révolus.

La Commission du Conseil des Etats avait mis au point un projet particulièrement pertinent en mettant sur pied d'égalité le financement de l'ambulatoire et l'hospitalier. De son côté, la référence hospitalière pour l'assurance de base serait le forfait DRG (diagnosis-related group), que le traitement ait lieu dans un établissement public ou privé pour autant que ce dernier figure sur la liste de planification. Les cantons ne l'entendirent pas de cette oreille et souhaitèrent garder l'entier de leurs prérogatives sous menace larvée de référendum. Personne ne les conteste, mais un peu d'esprit innovateur, s'il vous plaît! La Conférence des directeurs sanitaires n'a pourtant rien de mieux à proposer que le maintien du dispositif antérieur à la décision du Tribunal fédéral des assurances avec séparation rigoureuse du privé, entièrement à la charge des caisses-maladie, et de l'assurance de base valable uniquement dans les hôpitaux publics. Donc renvoi par le Conseil des Etats à sa Commission avec mandat d'entreprendre une négociation avec les cantons ...

Dans le même paquet figure aussi une amélioration du fonds de compensation entre les caisses («compensation des risques»), condition sine qua non pour mettre un terme à la chasse aux bons risques et à la création de caisses-maladie «bon marché».

Les caisses-maladie n'ont pas gagné en crédibilité dans cette opération. Elles ont cru pouvoir forcer le passage en nommant dans leurs conseils d'administration une multiplicité de parlementaires des deux Conseils, tous partis confondus. Le Conseiller fédéral Pascal Couchepin se voit lui-même soupçonné d'accointances particulières. Même si c'est à tort, certaines coïncidences poussent à le croire (offre du Groupe Mutuel en matière de médecine complémentaire $24 \mathrm{~h}$ après l'annonce de la fin de leur prise en charge par l'assurance de base). Une restructuration des caisses-maladie pour redonner aux assurés la voix qui leur manque est donc urgente. Si les conseils d'administration étaient élus par les assurés et non par copinage ou affinités tacticiennes machiavéliques, il en irait certainement autrement. Un système démocratique permettrait la circulation verticale de l'information permettant une meilleure compréhension des assurés, et réciproquement, aux directions une perception de leurs attente. Il valoriserait aussi la mission sociale de l'assurance obligatoire et sa qualité de partenaire.

De son côté, la FMH et ses membres se sont soumis à la neutralité des coûts pendant les 18 mois qui ont suivi l'introduction du nouveau tarif TARMED le $1^{\text {er }}$ janvier 2004. Cela a nécessité la mise en place d'un monitoring permanent avec des corrections de la valeur du point chaque fois que les coûts sont sortis du corridor de variations admissibles. Ce modèle, très efficace et couronné de succès, se voit relayé par un accord sur la gestion des coûts (LeiKoV) qui en reprend les modalités avec définition d'une cible négociée à respecter.

Pourquoi ce dispositif partenarial n'a-t-il pas été introduit aussi pour les autres secteurs de la santé, en particulier les hôpitaux, alors que leur responsabilité dans les augmentations constatées est particulièrement importante? Il devrait aussi être possible de mettre en place le même monitoring au niveau des médicaments, des soins à domicile et des EMS.

De toute évidence la volonté manque. Ce dispositif ne demande pourtant aucune modification fondamentale de la loi. Mais pourtant il faut agir vite, les uns et les autres, politiques compris, dans l'intérêt de toutes et tous. Le temps des combines et des luttes de pouvoir est terminé. Chacun a des compétences, et des revendications légitimes aussi. Il faut trouver des solutions ensemble, dans le respect mutuel, et travailler ensemble. Sinon ce sera la caisse unique que de nombreux médecins, excédés eux aussi, appellent de leur vœux ... A bon entendeur?

Dr Yves Guisan, vice-président de la FMH, Conseiller national 


\section{Prämienerhöhung bei den Krankenkassen: Die Toleranzschwelle ist erreicht!}

Das Karussell der Prämienerhöhungen, das jeden Herbst neu angestossen wird, hat diesmal Reaktionen hervorgerufen, die weit über die üblichen Unmutsbezeugungen hinausgehen. Eines ist sicher: Der Prozess der laufenden Prämienerhöhungen kann so nicht weitergehen. Die von politischer Seite vorgebrachten Ausflüchte und Blockaden sind unverständlich, und die Zeiten, in denen man sich gegenseitig den Schwarzen Peter zuschiebt, sind vorbei.

Die Kommission des Ständerats hat mit der Schaffung der Gleichheit bei der Finanzierung des ambulanten und des stationären Bereichs ein ausserordentlich sachdienliches Projekt erarbeitet. Andererseits wäre die DRG-Pauschale (diagnosis-related group) die Referenz der Spitäler für die Grundversicherung, unabhängig davon, ob die Behandlung in einem öffentlichen oder privaten Krankenhaus stattfindet, und vorausgesetzt, es steht auf der Spitalliste.

Die Kantone sehen dies aber anders und beharren unter Androhung eines Referendums auf ihren Vorrechten. Diese werden von niemandem bestritten, aber eine neue Betrachtungsweise könnte nicht schaden! Die Konferenz der Sanitätsdirektoren hat dagegen nichts anderes vorzuschlagen als die Beibehaltung des Zustands vor dem Beschluss des Eidgenössischen Versicherungsgerichtes - mit strikter Trennung zwischen dem Privatsektor, der zu Lasten der Krankenkassen geht, und der Grundversicherung, die nur für die öffentlichen Spitäler gilt. Dies bedeutet: erneuter Auftrag an die Ständeratskommission für Verhandlungen mit den Kantonen.

Im selben Paket findet sich auch ein verbesserter Ausgleichsfonds für die Kassen («Risikoausgleich»), der eine Conditio sine qua non dafür darstellt, dass die Jagd auf Mitglieder mit geringem Krankheitsrisiko und die Gründung «billiger» Krankenkassen aufhören.

Die Krankenkassen haben in diesem Spiel nicht an Glaubwürdigkeit gewonnen, weil sie durch Berufung von Parlamentariern aller Parteien in ihre Verwaltungsräte glauben, schneller ans Ziel gelangen zu können. Bundesrat Pascal Couchepin selbst sieht sich dabei einem bestimmten Verdacht ausgesetzt. Selbst wenn sich dieser nicht bestätigt, sprechen doch bestimmte Ereignisse dafür, so beispielsweise das Angebot der Groupe Mutuel bezüglich der Komplementärmedizin, das nur 24 Stunden nach der Ankün- digung erfolgte, dass diese Leistungen aus der Grundversicherung gestrichen werden. Deswegen ist eine Reorganisation der Krankenkassen, die den Versicherten die Stimme wiedergibt, die ihnen jetzt fehlt, dringend erforderlich. Wenn die Verwaltungsräte von den Versicherten und nicht von Klüngeln oder machiavellistischen und taktisch agierenden Seilschaften gewählt würden, liefen die Dinge anders. Ein demokratisches System mit vertikalem Informationsfluss würde den Versicherten Durchblick und den Direktionen Einblick in deren Erwartungen ermöglichen. Darüber hinaus würden die sozialen und partnerschaftlichen Aspekte der obligatorischen Krankenversicherung viel deutlicher.

Die FMH und ihre Mitglieder haben sich für Kostenneutralität während 18 Monaten nach Einführung der TARMED-Tarife am 1. Januar 2004 ausgesprochen. Dies machte eine ständige Überwachung und eine Korrektur der Taxpunktwerte nötig, wenn die Kosten den Toleranzbereich überschritten. Dieses Modell hat sich als sehr wirksam und erfolgreich erwiesen. Es wird nun durch eine Vereinbarung zur Kostenüberwachung (LeiKoV) abgelöst, das die Regeln mit der Festlegung eines Ziels verbindet, das es zu respektieren gilt.

Warum wurde eine solche partnerschaftliche Regelung nicht auch für andere Bereiche des Gesundheitswesens - insbesondere für die Spitäler - getroffen, die für die ständige Kostensteigerung besonders verantwortlich sind? Es sollte doch möglich sein, eine solche Kostenüberwachung auch bei Medikamenten, der Hauspflege und der Pflege von Heimbewohnerinnen und -bewohnern durchzuführen!

Ganz offensichtlich fehlt dazu der gute Wille. Eine grundlegende Gesetzesänderung ist dafür nämlich nicht nötig. Im Interesse aller muss aber schnell gehandelt werden - auch von den Politikern. Die Zeit der Manövertricks und Machtkämpfe ist vorüber. Gewiss hat jeder seine Kompetenzen und legitimen Ansprüche, es müssen aber gemeinsam und im gegenseitigen Respekt Lösungen erarbeitet werden. Andernfalls wird es die Einheitskrankenkasse geben, vor der viele auch empörte Ärzte - warnen.

Dr. med. Yves Guisan, Vizepräsident der FMH, Nationalrat 